\title{
Electromyographic Characterization in an Animal model of Dystonia
}

Kunal Dilip Chaniary

Virginia Commonwealth University

Follow this and additional works at: https://scholarscompass.vcu.edu/etd

Part of the Biomedical Engineering and Bioengineering Commons

(C) The Author

\section{Downloaded from}

https://scholarscompass.vcu.edu/etd/648

This Thesis is brought to you for free and open access by the Graduate School at VCU Scholars Compass. It has been accepted for inclusion in Theses and Dissertations by an authorized administrator of VCU Scholars Compass.

For more information, please contact libcompass@vcu.edu. 
(C) Kunal Dilip Chaniary 2008

All Rights Reserved 


\section{ELECTROMYOGRAPHIC CHARACTERIZATION IN AN ANIMAL MODEL OF DYSTONIA}

A thesis submitted in partial fulfillment of the requirements for the degree of Masters of Science at Virginia Commonwealth University.

by

KUNAL DILIP CHANIARY

Bachelors of Science, Mumbai University, India, 2005

Director: DR. PAUL A. WETZEL

ASSOCIATE PROFESSOR, DEPARTMENT OF BIOMEDICAL ENGINEERING

Virginia Commonwealth University

Richmond, Virginia

May 2008 


\section{Acknowledgement}

Firstly, I would like to thank my advisor, Dr. Wetzel for his guidance throughout this project and his encouragement in pursuing this exciting line of research. His insights to digital signal processing have helped me tremendously in this project.

I would like to express my gratitude to Dr. Shapiro for providing me with an opportunity to work on this research. His in-depth knowledge and logical way of thinking has been of great help to me. His extensive discussions around my work and constructive comments have been very helpful throughout this project.

I am extremely thankful to Dr. Mark Baron whose help, suggestions, patience and encouragement helped me at all times during this research project. His perpetual energy and enthusiasm in research has motivated me to work harder towards my research. Throughout the entire project he has encouraged me to develop independent thinking and research skills.

I would like to thank Dr. Ann Rice for all her invaluable suggestions and guidance throughout this project and for the efforts she has put in helping me understand the field of neuroscience better. Her detailed review, constructive criticism and excellent advice have helped me immensely in improving my skills for technical writing.

Last and most importantly, I would like to thank my parents for their enduring love and support throughout my life in achieving my dreams. None of this would have been possible without their blessings and encouragement. To them I dedicate this thesis. 
Also, I would like to thank my friends who have been a constant source of encouragement throughout my graduate study. Especially I am extremely thankful to Juni Sarkar as her care, support and valuable hints have helped me immensely to complete this project. 


\section{$\underline{\text { Table of Contents }}$}

Page

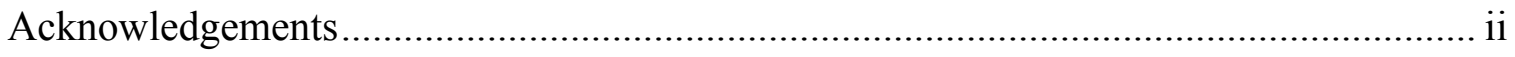

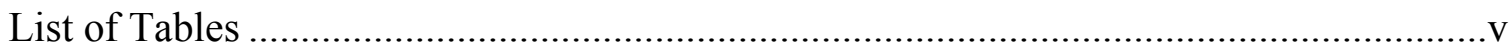

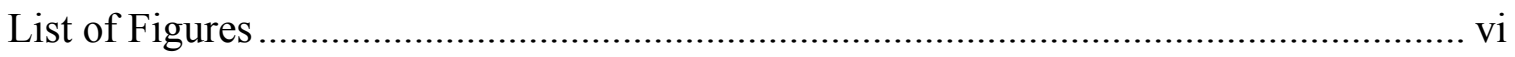

Chapter

1 Introduction .........................................................................................

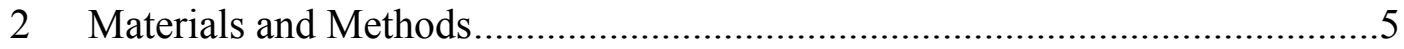

Groups and Clinical Assessments ...................................................

Recording of EMG activity .........................................................6

Decomposition of EMG signal into relevant frequency bands .................. 7

Power spectral density and coherence analysis ...................................10

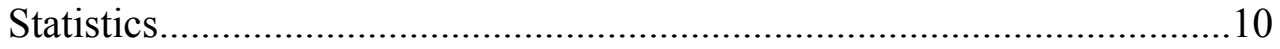

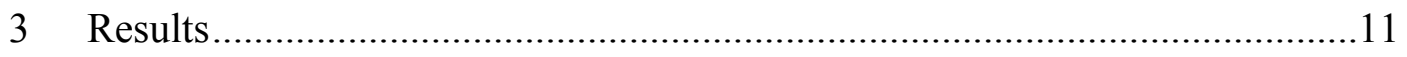

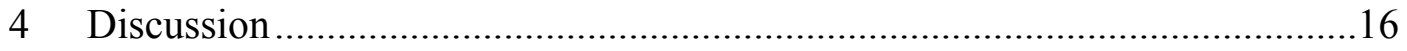

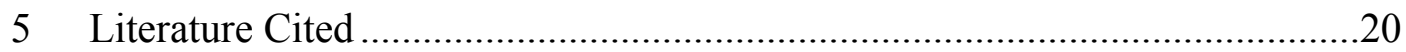




\section{$\underline{\text { List of Tables }}$}

Page

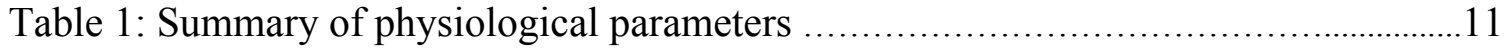




\section{$\underline{\text { List of Figures }}$}

Page

Figure 1:. Illustration of Wavelet shrinkage scheme .................................................

Figure 2: Representative decomposition of EMG signal ........................................

Figure 3: Electromyographic activity in normal and dystonic rats...............................12

Figure 4: Representative power spectral density distribution from a sulfa treated

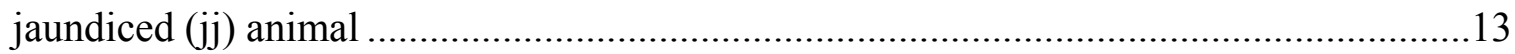

Figure 5: Percentage power spectral density in 4 experimental groups.......................... 14

Figure 6: Coherence spectra in four groups of animals ..........................................15 


\begin{abstract}
ELECTROMYOGRAPHIC CHARACTERIZATION IN AN ANIMAL MODEL OF DYSTONIA

By Kunal D Chaniary, M.S.
\end{abstract}

A Thesis submitted in partial fulfillment of the requirements for the degree of Masters of Science at Virginia Commonwealth University.

Virginia Commonwealth University, 2008

Director: Paul A. Wetzel, Ph.D.

Associate Professor, Department of Biomedical Engineering

Kernicterus causes damage to the auditory system and the basal ganglia in humans. Although the Gunn rat model of kernicterus has been extensively used to characterize the auditory features, this model has not been utilized to systematically investigate the movement disorder. In the present study, spontaneously jaundiced (jj) 16 day old Gunn rat pups were treated with sulfadimethoxine to exacerbate bilirubin neurotoxicity and compared to saline treated jjs and non-jaundiced $(\mathrm{Nj})$ littermates. Electromyographic (EMG) activity was recorded from antagonistic hip muscles in dystonic and in normal 
appearing rats. Raw EMG signals were decomposed using the Discrete Wavelet Transform based multi-resolution analysis, and signal coefficients corresponding to the dominant EMG frequency band were chosen. Gunn rats exposed to sulfadimethoxine developed a stable clinical state characterized by prolonged abnormal axial and appendicular postures. Coherence plots revealed 4-7 Hz co-activation in antagonistic muscles that was significantly more prominent in $\mathrm{jj}$ sulfa treated dystonic compared to normal rats. The EMG findings support the presence of dystonia in sulfadimethoxine exposed jj Gunn rats. 


\section{Introduction}

Dystonia is characterized by sustained, abnormal stereotyped posturing. ${ }^{1,2,3}$ The movement disorder is characterized physiologically by co-contractions of antagonistic muscles and overflow contraction of nearby muscles. ${ }^{2,4}$ Dystonia can be classified based on the age of onset, anatomical distribution, and etiology. ${ }^{3}$ Classification by anatomical distribution includes focal dystonia in which only a single body part is affected, segmental dystonia where two or more body parts are involved and generalized dystonia characterized by involvement of muscles throughout the body. ${ }^{5}$ Another way to classify dystonia is based on etiology, including primary dystonia describing inherited or sporadic cases and secondary dystonia, which, for example, can result from strokes or the effects of drugs. 5

Kernicterus or bilirubin encephalopathy, brain damage due to excessive neonatal jaundice (hyperbilirubinemia) involves damage to the brainstem auditory nuclei and the basal ganglia. ${ }^{6-8}$ Kernicterus is caused by high amounts of bilirubin in the infant's blood. Bilirubin a breakdown product of heme catabolism is lipid soluble, water insoluble and neurotoxic. Under normal circumstances, unconjugated bilirubin in the blood is bound to albumin and any extra bilirubin is conjugated in the liver by uridine diphosphate glucuronosyl transferase (UDPGT) and excreted in bile. However, in infants with

kernicterus, due to immaturity of glucuronosyl transferase the bilirubin binding capacity of 
serum albumin is exceeded and the levels of unconjugated-indirect bilirubin in the blood increases. This extra bilirubin crosses the blood-brain barrier and penetrates the brain causing permanent brain damage. Depending on the level of exposure, the effects range from unnoticeable to severe brain damage. The basal ganglia and the auditory system are most susceptible to damage from the bilirubin toxicity. Damage to the auditory system can lead to auditory neuropathy and under prolonged exposures can cause permanent hearing loss. Damage to the basal ganglia results in dystonia, athetosis and spasticity. The syndrome in term infants can be identified by high pitched cry, poor sucking or nursing, weakness, abnormalities of muscle tone including increased muscle tone (hypertonia) and decreased muscle tone (hypotonia), lethargy, arching of the back and spine, fever and abnormal auditory brainstem potentials. ${ }^{9}$

Permanent neurological damage in kernicterus can be prevented if detection and diagnosis is done at an early stage. Currently the clinical diagnosis in kernicterus can be confirmed using magnetic resonance imaging (MRI) and Brainstem Auditory Evoked Potentials (BAEPs). BAEP's measured in hyperbilirubinemic infants show an increase in interwave interval indicating increased conduction time and decrease in amplitude indicates auditory dys-synchrony. ${ }^{6-10}$ One of the most common method used for hyperbilirubinemia treatment is phototherapy. ${ }^{9,11}$ In this method light causes photoisomerization of bilirubin into non-toxic water soluble products thereby preventing toxic accumulation of bilirubin in the body to prevent permanent brain damage. Another method used to treat severe hyperbilirubinemia is exchange transfusion. ${ }^{9,11}$ In this therapy 
the baby's blood is replaced with donor's blood that does not contain bilirubin. The goal of this procedure is to remove enough bilirubin to prevent severe complication of brain damage due to kernicterus.

The jaundiced (jj) Gunn rat model is a well established model of bilirubin encephalopathy and kernicterus. ${ }^{12,13}$ This model provides an opportunity to study kernicterus and bilirubin toxicity in the laboratory and helps to understand the mechanisms, and pathophysiology that underlie this disorder. The homozygous recessive (jj) Gunn rat lacks the enzyme uridine diphosphate glucuronosyl transferase, which is responsible for the conversion of unconjugated bilirubin to conjugated bilirubin. ${ }^{12,13}$ In jj Gunn rats, the serum levels of total and unconjugated bilirubin peak at about postnatal day 16 and remain moderately elevated throughout the animal's life. Their usually mild bilirubin encephalopathy can be accentuated by injecting the jj rats with sulfadimethoxine (sulfa) on day 16 to displace bilirubin from blood albumin sites into tissues, including the brain. ${ }^{6,12-14}$ Within hours after sulfa injection, the rats develop auditory system dysfunction and abnormal prolonged axial and appendicular posturing, which closely resembles the clinical syndrome seen in human kernicterus. Heterozygous non-jaundiced $\mathrm{Nj}$ rats have about $50 \%$ of the normal enzyme activity and are phenotypically normal. ${ }^{14}$ The jj Gunn rat has many of the same clinical symptoms and histopathologic lesions that are exhibited by newborn suffering from kernicterus. 
Previous studies on the Gunn rat model have focused particularly on the damage in the auditory brainstem pathways. ${ }^{12-19}$ In contrast, the clinical aspects and the pathophysiological correlate of the movement disorder have never been systematically investigated in this animal model. The purpose of this study is to use electromyographic (EMG) recordings and coherence analysis to support our clinical impression that the movement disorder in jaundiced Gunn rats represents dystonia. These methods could also importantly be utilized to support the validity of other phenotypic animal models of dystonia. 


\section{MATERIALS AND METHODS}

All animals and procedures used for the study were approved by the Institutional Animal Care and Use Committee (IACUC) at Virginia Commonwealth University. A total of 32 animals were obtained from our in house breeding colony for this study. Animals from six different litters were used to account for inter-litter variability. The pups were weighed on day 16 and blood samples were drawn via a cheek puncture to determine hematocrit (Hct) and total plasma bilirubin (TB) levels.

\section{Groups and Clinical Assessments:}

A total of $18 \mathrm{jj}$ animals received an intraperitoneal injection of sulfa $(100 \mathrm{mg} / \mathrm{kg})$ on day 16. Five of these animals developed mild-to-moderate motor disability and comprised the experimental group. Of the remaining animals, six were severely affected and did not survive the acute bilirubin encephalopathy, and seven were not affected and were not studied further. Control groups consisted of $\mathrm{jj}$ s given saline $(\mathrm{n}=5)$ and $\mathrm{Njs}$

given sulfa $(n=5)$ or no treatment $(n=4)$. Littermate jjs and Njs were randomly assigned to either experimental sulfa or control groups. All animals were carefully monitored each day for loss of body weight, which was compensated with an oral feeding of kitten milk formula or a subcutaneous injection of $5 \%$ dextrose in $0.45 \% \mathrm{NaCl}$ or a combination of both. Animals were clinically examined daily, and a clinical score (CS) between 0 to 5 was assigned based on the severity of the movement disorder ( 0 - normal, 1- slight limb 
dystonia \& gait abnormality, 2-mild limb dystonia \& gait abnormality, and impaired righting reflex, 3- moderate limb dystonia and gait abnormality, with prolonged righting reflex, 4- severe failure of ambulation, general lack of spontaneous movement with occasional bursts of hyperactivity, and no righting reflex, and 5- moribund, including

seizures and agonal respiration), ${ }^{19}$ and 0.5 was added to the score of animals appearing to be midway between a category and the next higher one. The five dystonic jj sulfa animals used in this study received scores between 1.0 and 2.5. Six dystonic jj sulfa animals given scores $\geq 3.5$, two each at 3.5, 4.0 and 4.5, became severely dystonic and did not survive to 34 days of age; seven additional jj sulfa animals, all receiving scores of 0.5 , were clinically unaffected and not used for the study. Thus, for this study, the scale functionally ranges from 0 to 4 .

\section{Recording of EMG Activity:}

EMG activity was recorded on day 34, more than two weeks after sulfa injection. We targeted day 34 as an optimal age for recording EMG activity to allow sufficient time for the animal to recover from the acute bilirubin encephalopathy and for their neurological symptoms to stabilize, and for the animal to grow larger, allowing easier targeting of muscles for percutaneous insertion of fine wire electrodes.

On the day of recording, the lower backs of the rats were shaved to allow targeting of the muscles for electrode insertion. Rats were lightly anesthetized with an intramuscular injection of ketamine $(30 \mathrm{mg} / \mathrm{kg})$ and acepromazine $(3 \mathrm{mg} / \mathrm{kg})$. Throughout the recording, the body temperature was monitored with a rectal temperature probe and 
held at $37^{0} \pm 0.1^{0} \mathrm{C}$ with a heating pad. Teflon coated $50 \mu \mathrm{m}$ stainless steel fine wire electrodes (A-M systems, Carlsborg, WA) were inserted percutaneously into antagonistic hip muscles, the gluteus superficialis (hip flexion) and the gluteus medius (hip extension) via a 30 gauge needle. Muscles were stimulated electrically (Grass Technologies, West Warwick, RI) and the hip motion was observed in order to verify correct placement of the electrodes. Recordings were assessed as the animals awakened from anesthesia. In the partially sedated state, a small pinch was applied to the toes and EMG activity was recorded in response to this stimulus. Multiple such stimuli were applied and EMG activity was recorded for a minimum of at least $120 \mathrm{sec}$. The signals were amplified ( $\mathrm{x}$ $1000)$ and filtered $(10 \mathrm{~Hz}-1 \mathrm{KHz})$ through a differential AC amplifier (A-M Systems, Carlsborg, WA) and digitized through a NI-DAQ card (National Instruments Co., Austin, TX) at a sampling frequency $=4 \mathrm{KHz}$. EMG activity was monitored continuously on a desktop computer using Sort Client (Plexon Inc., Dallas, TX).

\section{Decomposition of the EMG Signals into Relevant Frequency Bands:}

The raw EMG signal in dystonia consists of repetitive bursts superimposed on sustained hypertonic activity. ${ }^{20,21}$ For accurate estimation of coherence between antagonist muscle pairs, it is necessary to separate the high frequency bursts from sustained hypertonic muscle activity. ${ }^{20,21}$ An effective way to achieve this separation is to decompose the EMG signal using the Discrete Wavelet Transform (DWT) based multiresolution approach. ${ }^{20-24}$ The DWT approach involves passing the EMG signals progressively through a series of filters with different cutoff frequencies; thereby,

decomposing the original signals into different frequency bands. ${ }^{23,24}$ 


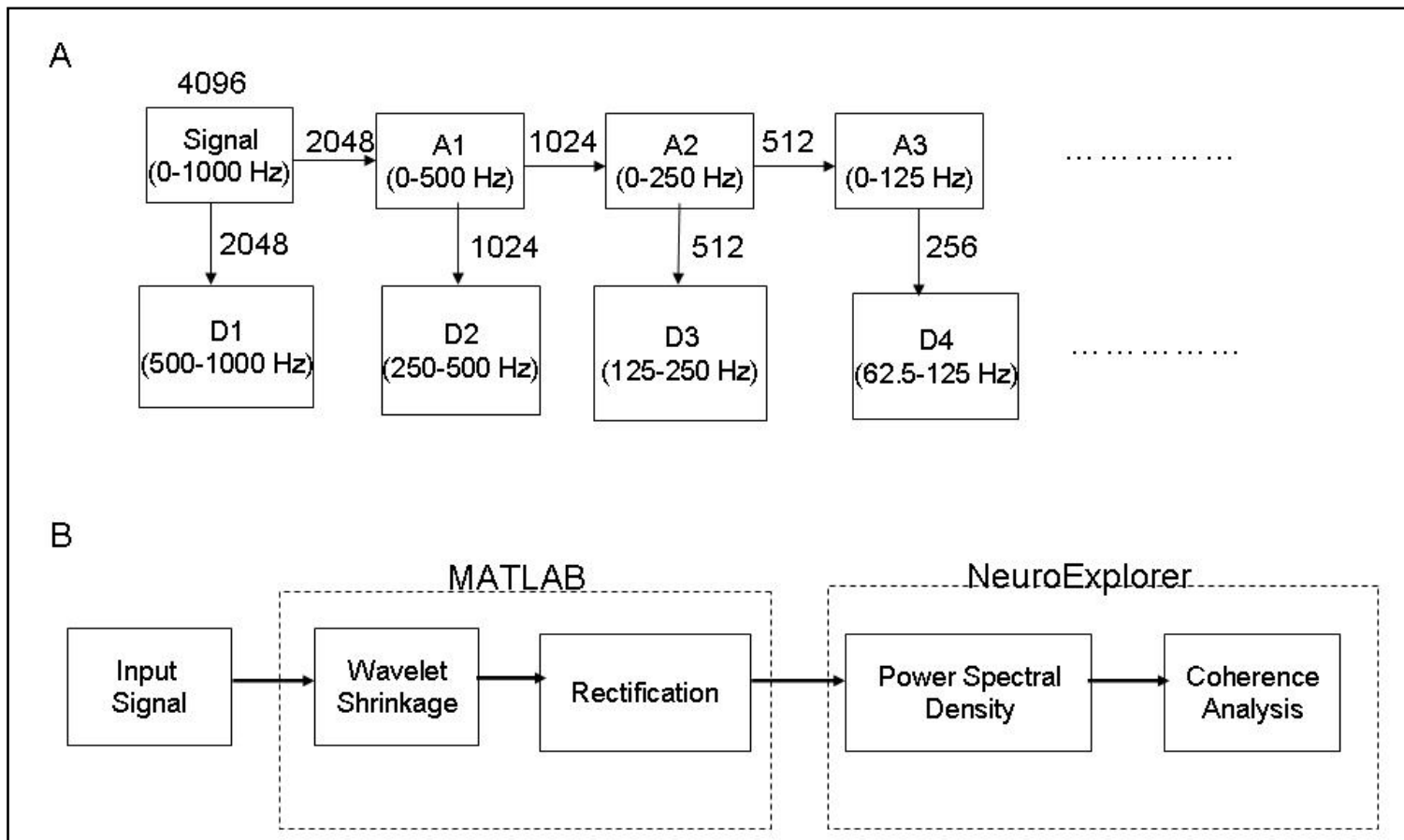

Figure 1: A. Illustration of the Wavelet shrinkage scheme. An input signal with bandwidth of 0$1000 \mathrm{~Hz}$ is filtered and downsampled into low frequency (A1, A2...) and high frequency (D1, D2...) components. B. Block diagram representation of analysis procedure for Electromyographic (EMG) signals.

At each level of decomposition, the raw EMG signal is decomposed into approximate (A1, A2...) and detail (D1, D2 ...) coefficients (Fig. 1A). The approximate coefficients are the high scale, low frequency components of the signal obtained by passing the original signal through a highpass scaling filter and downsampling by two. The detail coefficients are the low scale, high frequency coefficients of the signal obtained by passing the original signal through a lowpass scaling filter and downsampling by two. The high pass and low pass filters at each stage are related to each other and are called as quadrature mirror filters. The advantage of using a DWT analysis is that the time localization of frequencies is not lost during the decomposition of the 
signal. ${ }^{24}$ Because bursts in EMG signals are dominant in the $50-150 \mathrm{~Hz}$ frequency range, ${ }^{20,21}$ we subjected the raw signals to level eight decomposition, which permitted us to investigate the pattern of EMG-EMG coherence in $125-250 \mathrm{~Hz}$ (D3), $62.5-125 \mathrm{~Hz}$ (D4), and $31.25-62.5 \mathrm{~Hz}$ (D5) frequency bands. The EMG signals were decomposed and then rectified using Matlab (MathWorks Inc., Natick, MA). Power spectral and coherence analyses were performed using Neuroexplorer (Nex Technologies, Littleton, MA) (Fig 1B).A representative decomposition of an EMG signal using Discrete Wavelet Transform is shown in Fig. 2.
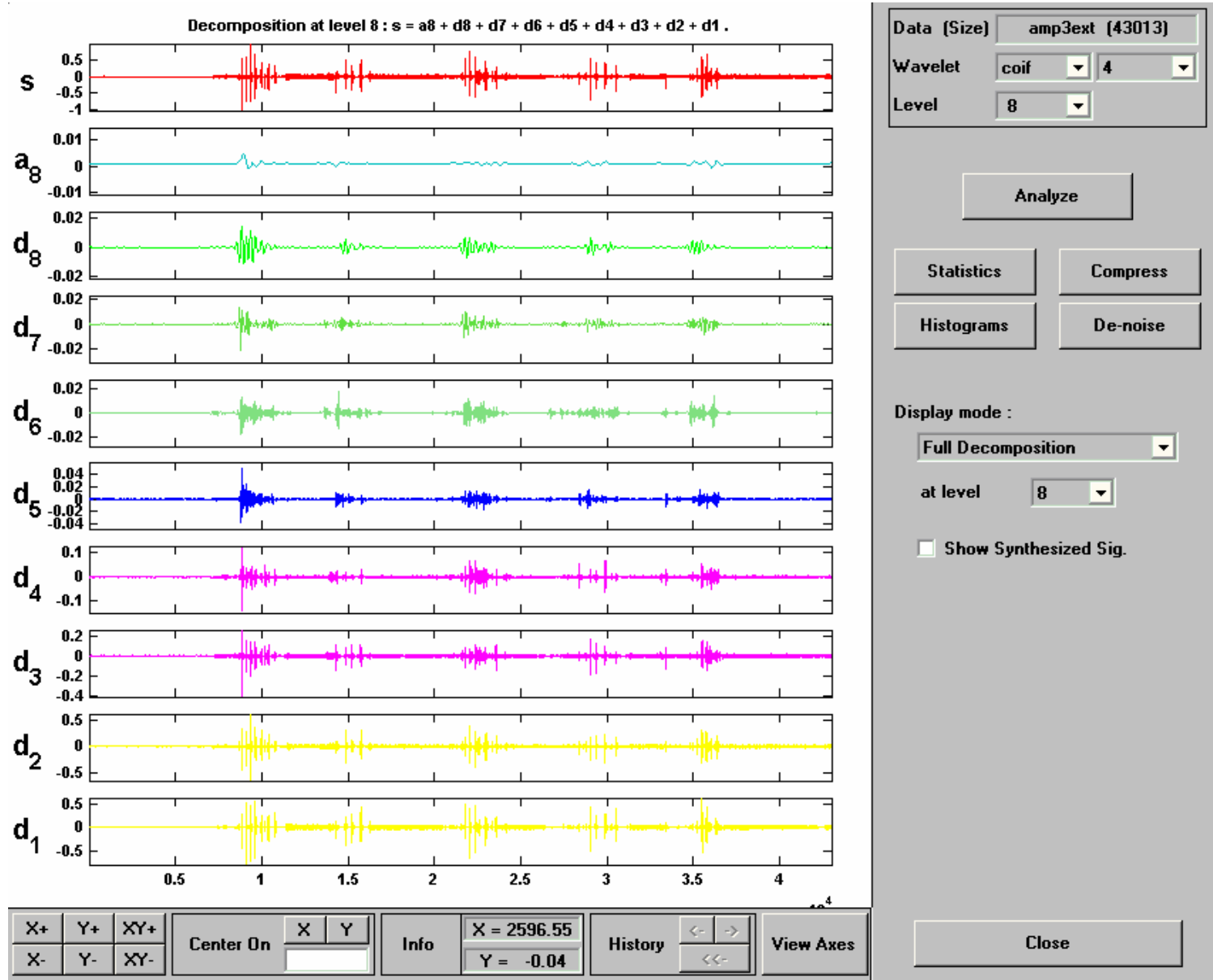

Figure 2: Representative decomposition of an EMG signal by Discrete Wavelet Transform (DWT) using MATLAB. d1-d8 are the detail coefficients and a8 is approximate coefficient. 


\section{Power Spectral Density and Coherence Analysis:}

Power spectral density is the measure of the average power in a signal per unit of frequency as a function of frequency. ${ }^{25}$ Coherence provides a measure of how similar two signals are and the linear association between them. ${ }^{2,26}$ Based upon the degree of association, coherence can be assigned values from 0 to 1 in which 0 corresponds to minimal or no coherence and 1 indicates maximal coherence. The coherence estimation of two signals a, b can be represented as

$$
\lambda_{a b}(f)=\frac{C_{a b}(f)}{\sqrt{C_{a a}(f) * C_{b b}(f)}}
$$

$\mathrm{C}_{\mathrm{ab}}(\mathrm{f})=$ cross spectral density of the two signals, and

$\mathrm{C}_{\mathrm{aa}}(\mathrm{f}), \mathrm{C}_{\mathrm{bb}}(\mathrm{f})=$ auto-spectra of each signal.

The data were smoothed by using a moving average filter (width $=1 \mathrm{sec}$ ) and coherence measures were obtained across multiple windows of one second duration along the entire length of each signal.

\section{Statistics:}

Statistical analyses were carried out by the one way analysis of variance (ANOVA) for multiple comparisons followed by Tukey-Kramer honestly significant difference (HSD) test. A probability value of $<0.05$ was considered to be statistically significant for making comparisons between groups. 


\section{RESULTS}

Table 1 provides a summary of physiological parameters and clinical scores (CS) for the animals undergoing EMG analysis. Gender was evenly distributed between the $\mathrm{jj}$ and $\mathrm{Nj}$ groups. Sulfa treated $\mathrm{jj}$ animals weighed significantly less than the animals in the other three groups at 34 days of age $(\boldsymbol{p}<0.001)$. Fig. 3A depicts a control animal and Fig. 3B represents the movement disorder seen in sulfa treated $\mathrm{jj}$ rats, characterized by sustained abnormal postures in the trunk and limbs. In sulfa treated $\mathrm{jj}$ animals, gait and balance were also affected in proportion to the degree of abnormal posturing.

Table 1: Summary of physiological parameters and clinical scores of animals used for Electromyographic (EMG) characterization.

\begin{tabular}{|l|r|r|r|r|r|}
\hline \multicolumn{3}{|c|}{ Day 16 } & \multicolumn{2}{c|}{ Day 34 } \\
\hline Group & Weight(gms) & TB(mg/dL) & CS & Weight $($ gms $)$ & CS \\
\hline jj sulfa $(n=5)$ & $26.1 \pm 2.7$ & $9.8 \pm 0.8$ & $0.5 \pm 0$ & $61 \pm 5.5$ & $1.5 \pm 0.5$ \\
\hline jj saline $(n=5)$ & $27.8 \pm 2.2$ & $10.33 \pm 1.4$ & $0.5 \pm 0$ & $79.8 \pm 7.6$ & $0.5 \pm 0$ \\
\hline Nj sulfa $(n=5)$ & $27.6 \pm 4.4$ & $0 \pm 0$ & $0 \pm 0$ & $87.5 \pm 5.0$ & $0 \pm 0$ \\
\hline Nj untreated $(n=4)$ & - & - & - & $107.5 \pm 10.7$ & $0 \pm 0$ \\
\hline
\end{tabular}

(TB- Total Bilirubin, CS- Clinical Scores)

Under light anesthesia, a tail pinch produced clear volitional movements typically lasting 10-15 seconds. In sulfa-treated animals, these movements closely resembled the abnormal spontaneous motor activity observed in unanesthetized dystonic animals and clearly differed from the movements induced in lightly anesthetized control animals. Subsequent to a tail pinch, EMG activity in all $\mathrm{Nj}$ and saline treated $\mathrm{jj}$ animals showed a pattern dominated by alternating activation of agonist and antagonist hip muscles.

Representative EMG activity in a sulfa treated $\mathrm{Nj}$ animal is shown in Fig $1 \mathrm{C}$. In contrast, 
co-contractions were regularly seen in agonist and antagonistic hip muscles in sulfa treated jj animals (Fig 1D).
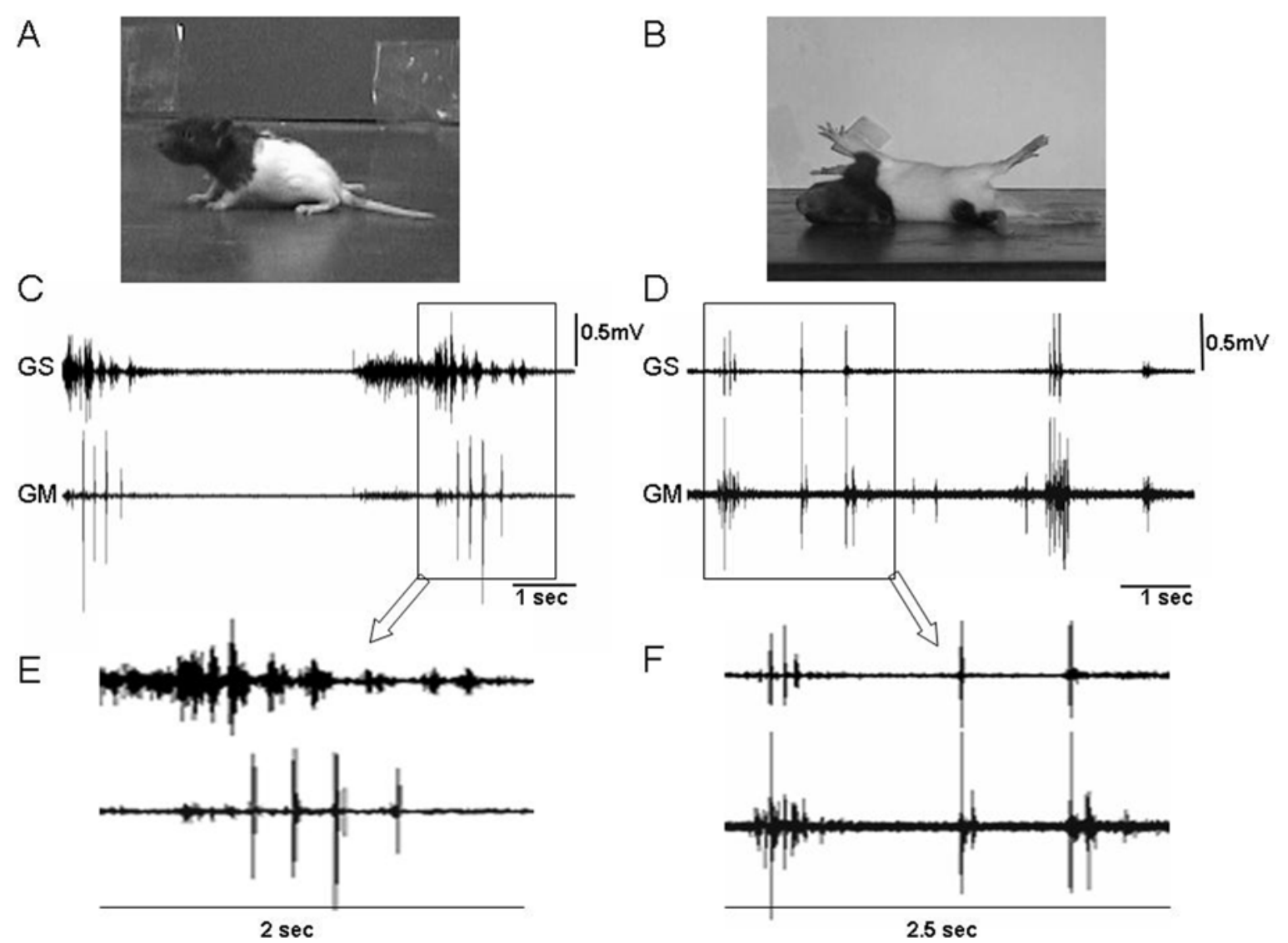

Figure 3: A. Normal nonjaundiced $(\mathrm{Nj})$ rat 24 hours after sulfa treatment B. Dystonic posture in a jaundiced (jj) rat 24 hours after sulfa treatment. Note the abnormal dystonic posture, especially in the hindlimbs C. Raw Electromyographic (EMG) activity recorded in the $\mathrm{Nj}$ rat shows alternating pattern of activation in antagonistic hip muscles (gluteus superficialis [GS], gluteus medius [GM]) D. Raw EMG activity in jj rat shows co-contractions in antagonistic hip muscles. E, F. Magnified view of EMG activity in C, D, respectively.

In sulfa treated jj animals, EMG activity showed a prominent peak in the power spectral density at $4.5 \mathrm{~Hz}$ in D3 signals and at $5 \mathrm{~Hz}$ in D4 signals (Fig 4). In D5 signals, a peak in power spectral density response is seen at greater than $8 \mathrm{~Hz}$. These frequency ranges however are not relevant to studies of dystonia ${ }^{2}$, and hence they have not been 
considered for further analysis. Of note, the peak response shifted towards higher frequencies with the increasing levels of decomposition (i.e. from D3 to D5).

\section{Power Spectral Densities}

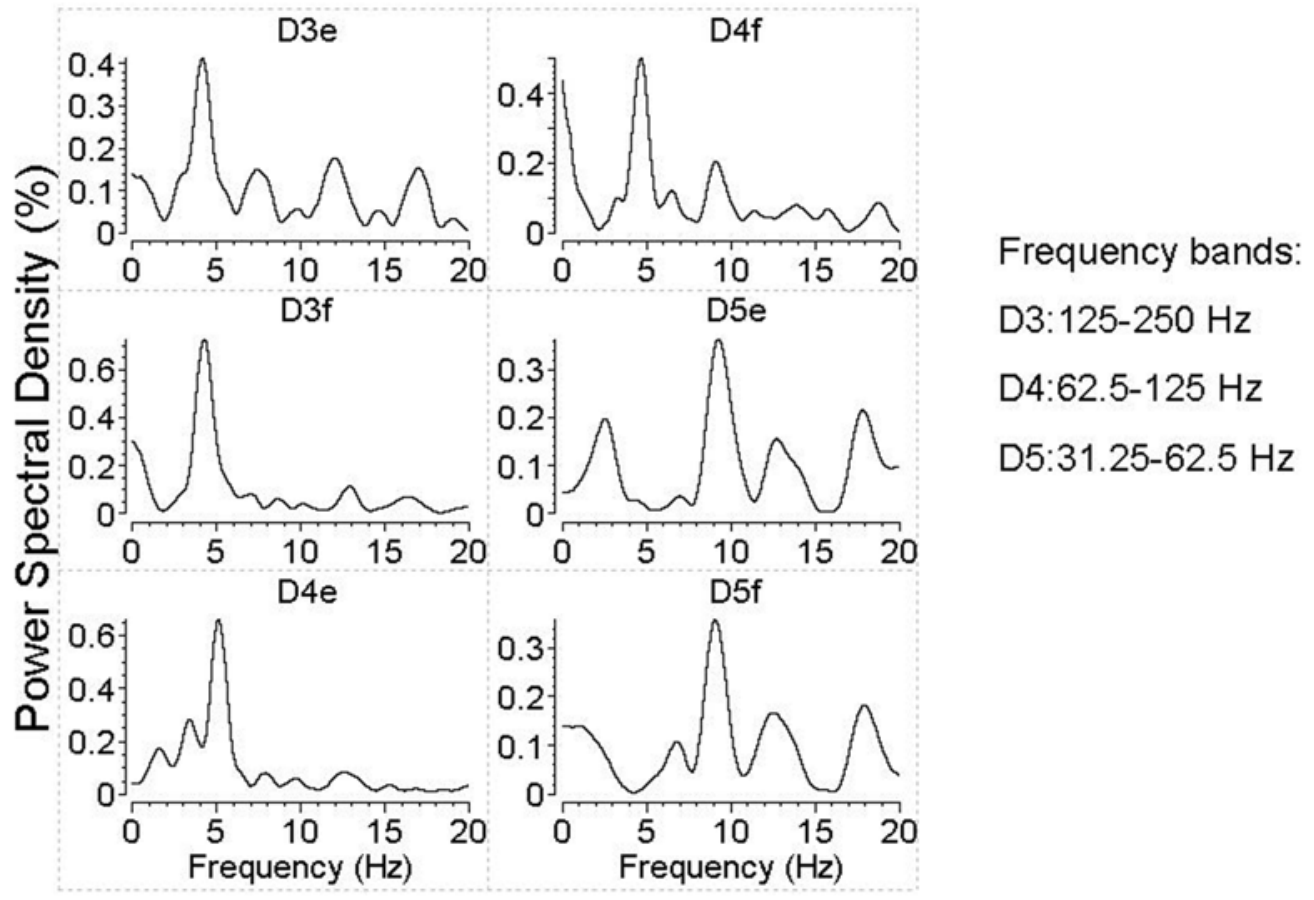

Figure 4: Representative power spectral density distribution from a sulfa treated jaundiced (jj) animal. Peak in the spectral density response can be seen at $4.5 \mathrm{~Hz}$ in D3 $(125-250 \mathrm{~Hz})$ signals and at $5 \mathrm{~Hz}$ in D4 (62.5$125 \mathrm{~Hz}$ ) signals for extensor (e) and flexor (f) muscles.

To compare groups, we calculated the average power spectra obtained in the four different groups of animals. The 4-7 Hz peak in power spectra was significantly prominent in D3 and D4 signals among the sulfa treated jj animals as compared to the other three groups (Fig 5). To compare the PSD's in the 4-7 Hz range in the four different groups of animals the unequal variance $F$ test was used. The F value for signals D3e, D3f, D4e, and 
D4f were $161.7,143.2,40.25,9.5$ respectively among the four groups of animals (all $\boldsymbol{p}$ values $<0.001$ ). Post hoc tests revealed that in all the 4 signals (i.e D3e, D3f, D4e, D4f) the jj sulfa group was significantly different that the other 3 groups (Tukey's HSD).
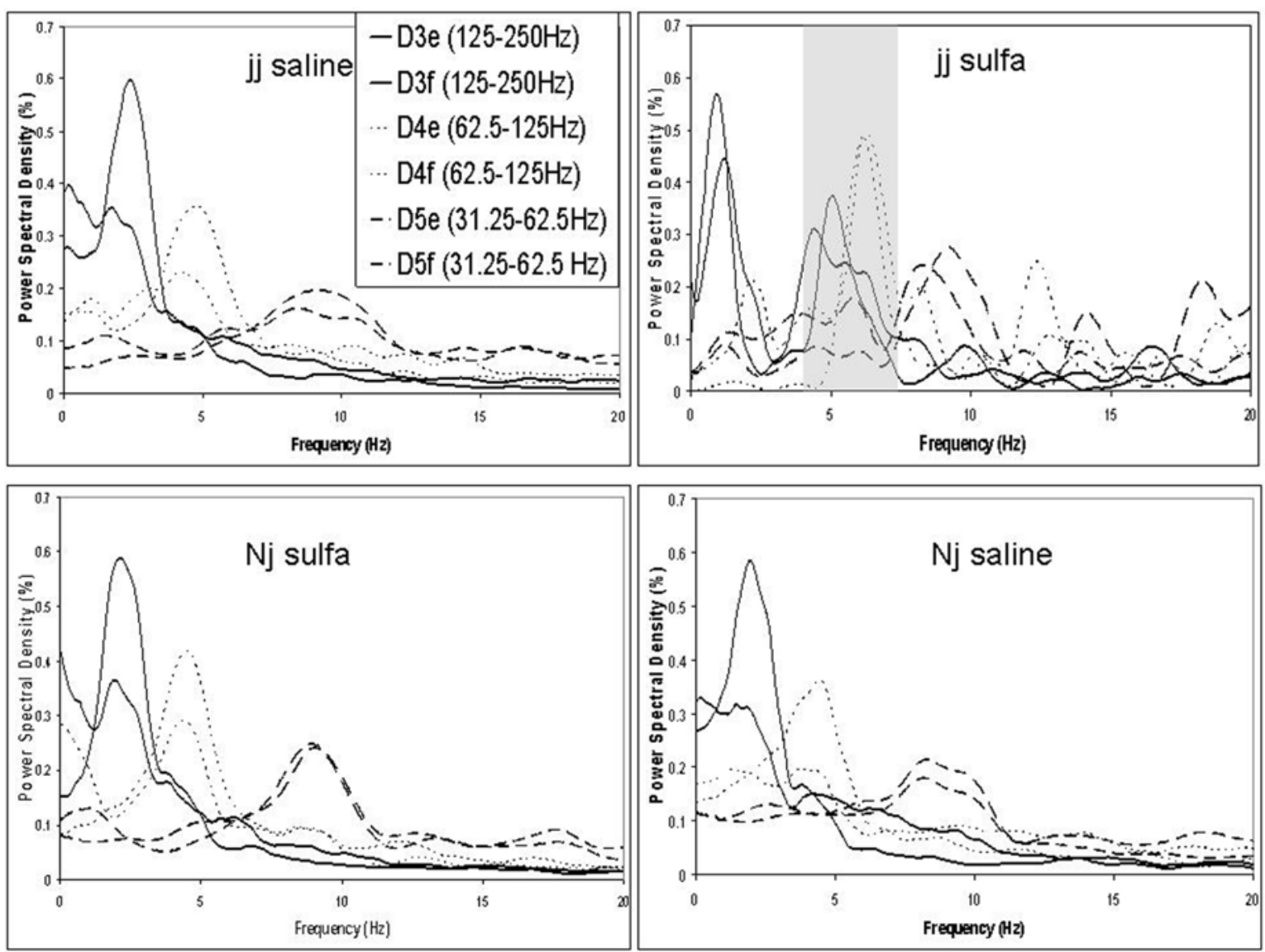

Figure 5: Percentage power spectral density in 4 experimental groups. There is an increase in the power spectra in 4-7 Hz range (highlighted area) in jaundiced (jj) sulfa treated animals in D3 (125-250 Hz) and D4 (62.5-125 Hz) signals for extensor (e) and flexor (f) muscles.

To look for antagonist muscle co-activation, we examined the coherence spectra in the hip muscle pairs among the four different groups of animals. Only sulfa treated $\mathrm{jj}$ animals showed an abnormal, prominent peak in the coherence spectra in the 4-7 Hz range (Fig 6A). The coherence for the D3 and D4 signals at 4-7 Hz were compared across the 
four groups using the unequal variance F-test and were found to be different (D3, F[3, $324.6]=3049.3 ; \mathrm{D} 4, \mathrm{~F}[3,325.77]=2802.2$; both p-values $<0.0001)($ Fig 6B). Using Tukey's HSD, sulfa treated jj animals (D3 mean $=0.796$, D4=0.789) were significantly different from the other three groups. There were no significant differences in coherence between the other three groups (D3 combined mean=0.433, D4=0.461), and there was no significant correlation between the power spectral density of the D3 and D4 signals and the dystonia rating of the rat.

A

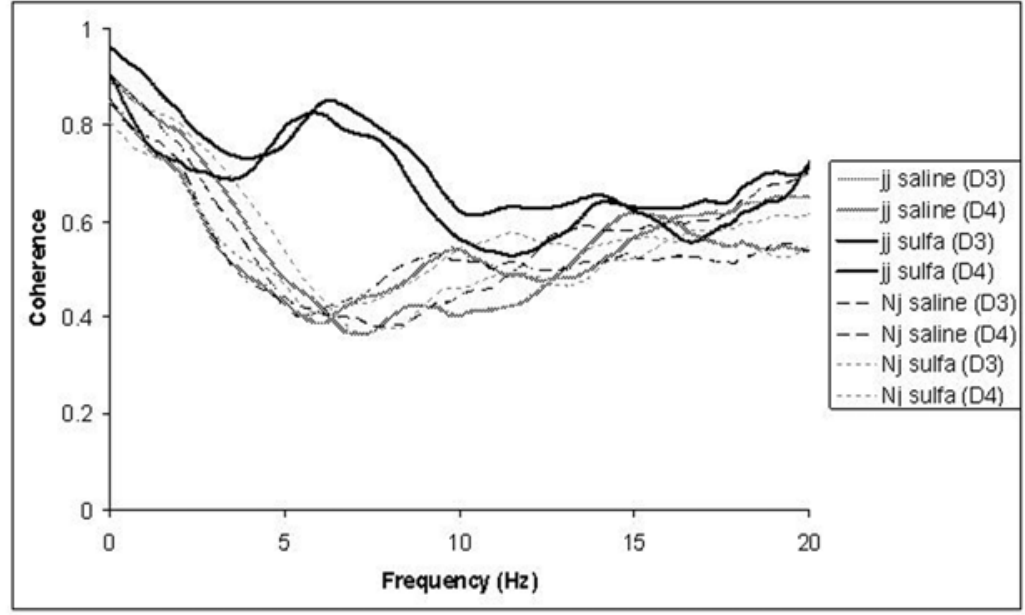

B

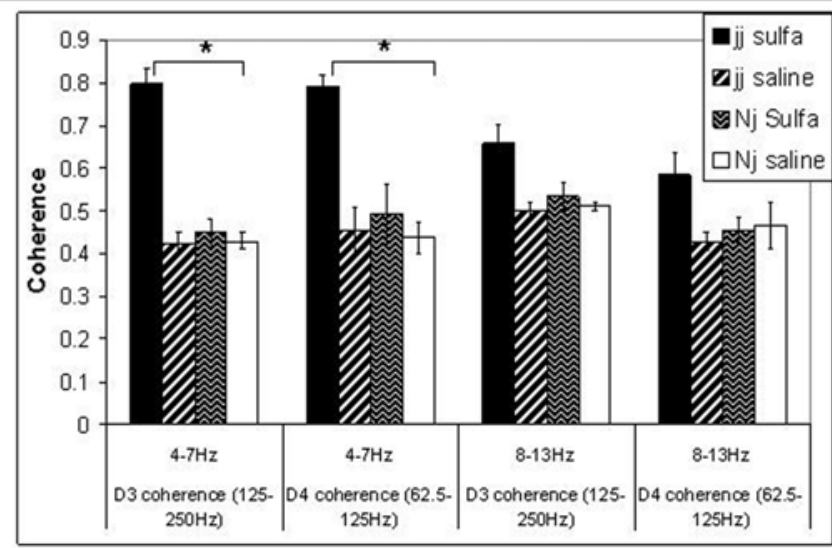

Figure 6: A. Coherence spectra of the D3 $(125-250 \mathrm{~Hz})$ and $\mathrm{D} 4(62.5-125 \mathrm{~Hz})$ signals in the 4 groups of animals. A clear prominent peak in the coherence spectra for antagonistic muscles can be seen in sulfa treated $\mathrm{jj}$ animals in the $4-7 \mathrm{~Hz}$ range B. Summary of coherence in the 4 groups $(* \mathrm{P}<0.05)$. Error bars indicate the standard error of the mean. 


\section{DISCUSSION}

In this study, EMG recordings from antagonistic hip muscle pairs revealed prominent co-contractions in impaired, but not control rats, supporting our clinical impression that jaundiced (jj) Gunn rats with kernicterus develop dystonia. Using the DWT methodology to enhance the separation of burst from background tonic activity, an abnormal 4-7 Hz drive was observed in antagonistic hip muscles in dystonic jj rats. With this methodology, a distinct 4-7 Hz peak in the PSD was shown more prominently in 125$250 \mathrm{~Hz}$ and $62.5-125 \mathrm{~Hz}$ signals, which encompass the usual EMG burst frequency band ranges. Prior studies in humans with dystonia have demonstrated a comparable 4-7 Hz drive in antagonistic muscle pairs thereby further supporting the validity of the jaundiced Gunn rat as a model for studying human dystonia. Moreover, because similar 4-7 Hz cocontraction drives have been established in primary human dystonias, studies in the Gunn rat model may have direct implications for primary as well as secondary human dystonias.

The present findings, along with findings from human studies of dystonia, suggest that a central 4-7 Hz drive to dystonic muscle pairs underlies the pathophysiology for many common forms of dystonia. Tijssen ${ }^{27}$ showed an abnormal synchronizing drive of 5$7 \mathrm{~Hz}$ in antagonistic cervical muscle pairs during involuntary contraction in patients with cervical dystonia. Grosse ${ }^{2}$ examined the differences in EMG discharge patterns in different etiological groups of patients with dystonia, and observed an abnormal synchronizing drive of 4-7 Hz in patients with primary dystonia. Wang ${ }^{21}$ studied the functional coupling 
between EMG's and local field potentials (FP) of the internal globus pallidum (GPi) in a patient with dystonia. A strong coherence was reported between the activity in the GPi and the muscles of the upper arm at frequencies of $4-7 \mathrm{~Hz}$ in the dystonic patient.

To our knowledge, this is the first time that co-contractions have been observed in antagonistic muscles in secondary dystonia. Grosse and his colleagues ${ }^{2}$ found an abnormal pattern of synchronizing descending drive of 4-7 Hz only in the distal lower limb of symptomatic patients with DYTI gene mutation (i.e. primary dystonia) and not in patients with secondary dystonias. One possibility to explain the discrepancy may be that many secondary dystonias do not have the 4-7 Hz drive in antagonistic muscle pairs. Another possibility could be that our Gunn rat model, despite being a model of secondary dystonia, may have pathophysiological aspects that are more typical of primary dystonias.

Other animal models have previously been introduced to investigate the pathophysiological aspects of dystonia. However though, to our knowledge, the presence of dystonia has never been well characterized via EMG studies in these models. The dystonic $d t^{s z}$ (sz-eplileptic seizures) hamster model shows a close resemblance to generalized paroxysmal dystonia in humans and has been used extensively to investigate neurophysiological correlates of basal ganglia in dystonia. ${ }^{5,28}$ Another animal model of generalized dystonia is the $d t$ rat. Expression of dystonia in these rats has been linked to the biochemical abnormalities within their cerebellum. ${ }^{29}$ Byl and colleagues ${ }^{30}$ have introduced a chronic model of focal hand dystonia in owl monkeys produced by repetitive 
hand opening and closing. The focal feature of this model offers an advantage of producing less distress to the animals than many chronic models, though its utility is also somewhat limited by this feature. In monkeys, dystonia has been produced by administration of various toxins including 4-phenyl-1,2,3,6-tetrahydropyridine (MPTP) and 3-nitropropionic acid (3-NP). These agents however only inconsistently induce dystonia and cannot be targeted effectively towards milder symptoms. ${ }^{31}$

The present study has a number of shortcomings. Firstly, the limited range of severity of the dystonia prevented us from assessing for possible correlations between EMG power spectral density of the D3 and D4 signals and the severity of dystonia. However, the lack of correlation suggests that wavelet analysis may be relatively more sensitive to dystonia than more traditional approaches. Secondly, the significance of our findings depends largely on the premise that co-contractions support a clinical impression of dystonia. Yet, co-contractions however can occur naturally in humans, for instance, during isometric exercise ${ }^{32}$ and moreover, dystonia may be exhibited with little or no coincident antagonist muscle involvement. ${ }^{33}$ Despite these caveats, we contend that the present demonstration of a 4-7 Hz co-contraction drive, comparable to that established for human dystonias and limited to animals exhibiting clinical dystonia, is strong evidence in support of the presence of clinically relevant dystonia. Finally, although the animals appeared alert and displayed their naturally dystonic or normal clinical states during the EMG data collection periods, our recording conditions imposed the limitations, especially the possible effects of anesthesia on the responses. However a clear difference in findings 
between our experimental and control groups argue that the effect is not due to anesthesia or other experimental conditions, which would have affected all groups equally. Future studies, are necessary to exclude the possibility that alert rats, moving naturally would, for example, exhibit a different pattern of EMG activity or would exhibit a stronger coherence pattern than lightly anesthetized, stimulated animals.

In summary, the present findings suggest that EMG may provide an objective means to verify the presence of dystonia in our animal model. This approach may provide a convenient and reliable means to objectively quantify dystonia for further experiments in the Gunn rat, as well as in other animal models of dystonia. The jaundiced Gunn rat model of kernicterus should serve as a reliable model to investigate pathophysiological mechanisms for dystonia, and to test novel therapies. 
$\underline{\text { Literature Cited }}$ 


\section{$\underline{\text { Literature Cited }}$}

1. Geyer HL, Bressman SB. The diagnosis of dystonia. Lancet Neurol 2006; 5: 78090.

2. Grosse P, Edwards M, Tijssen MAJ, Schrag A, Lees AJ, Bhatia KP, Brown P. Pattern of EMG-EMG coherence in limb dystonia. Mov Disord 2004; 19: 758-769.

3. Aguiar P, Ozelius L. Classification and genetics of dystonia. Lancet Neurol 2002; 1: 316-25.

4. Yanagisawa N, Goto A. Dystonia musculorum deformans. Analysis with electromyography. J Neurol Sci 1971; 13: 39-65.

5. Raike RS, Jinnah HA, Hess EJ. Animal models of generalized dystonia. NeuroRx 2005; 2 : 504-12.

6. Ahlfors C, Shapiro SM. Auditory brainstem response and unbound bilirubin in jaundiced (jj) Gunn rat pups. Biol Neonate 2001; 80: 158-62.

7. Shapiro SM, Hecox KE. Development of brainstem auditory evoked potentials in heterozygous and homozygous jaundiced Gunn rats. Dev Brain Research 1988; 41: $147-57$.

8. Shapiro SM. Reversible brainstem auditory evoked potential abnormalities in jaundiced Gunn rats given sulfonamide. Pediatr Res 1992; 34: 629-633.

9. Shapiro SM. Bilirubin toxicity in the developing nervous system. Pediatr Neurol 2003; 29: 410-21.

10. Shapiro SM, Nakamura H. Bilirubin and the auditory system. J of Perinatol 2001; 21: S52-S55.

11. Practice parameter: management of hyperbilirubinemia in the healthy term newborn. American Academy of Pediatrics. Provisional Committee for Quality Improvemtn and Subcommittee on Hyperbilirubinemia. Pediatrics, 1994. 94(4 Pt 1): $558-65$ 
12. Shapiro SM. Brainstem auditory evoked potentials in an experimental model of bilirubin neurotoxicity. Clin Pediatr 1994; 33: 460-67.

13. Shapiro SM. Somatosensory and Brainstem auditory evoked potentials in the gunn rat model of acute bilirubin neurotoxicity. Pediatr Res 2002; 52: 1-6.

14. Strebel L, Odell GB. Bilirubin uridine diphosphoglucuronyltransferase in rat liver microsomes: genetic variation and maturation. Pediatr Res 1971; 5: 548-59

15. Shapiro SM, Hecox KE. Developmental studies of brainstem auditory evoked potentials in jaundiced Gunn rats. Brain Res. 1988; 469: 147-57.

16. Shapiro SM, Hecox KE. Brainstem auditory evoked potentials in jaundiced Gunn rats. Ann Otol Rhinol Laryngol 1989; 98: 308-17.

17. Shapiro SM, Conlee JW. Brainstem auditory evoked potentials correlate with morphological changes in Gunn rat pups. Hear Res 1991; 57: 16-22.

18. Conlee JW, Shapiro SM. Morphological changes in the cochlear nucleus and nucleus of the trapezoid body in Gunn rat pups. Hear Res 1991; 57: 23-30.

19. Shaia W, Shapiro SM. Immunhistochemical localization of calcium-binding proteins in the brainstem vestibular nuclei of the jaundiced Gunn rat. Hear Res 2002; 173: 82-90.

20. Wang SY, Liu X, Yianni J, Aziz TZ, Stein JF. Extracting burst and tonic components from surface electromyograms in dystonia using adaptive wavelet shrinkage. J Neurosci Methods 2004; 139: 177-84.

21. Wang SY, Liu X, Yianni J, Tailor JK, Aziz TZ, Stein JF.Detecting functional coupling between electromyogram and local field potentials in dystonia using discrete wavelet transform and coherence estimation. Proceedings of the $1^{\text {st }}$ International IEEE EMBS 2003.

22. Reaz MBI., Hussain MS, Yasin F. Techniques of EMG signal analysis: detection, processing, classification and applications. Biol Proced Online 2006; 8: 11-35.

23. Mallat SG. A theory for multiresolution signal decomposition: the wavelet representation. IEEE Trans Pattern Anal Mach Intell 1989; 11: 674-93.

24. Polikar R. The Wavelet Tutorial http://users.rowan.edu/ polikar/WAVELETS/ WTtutorial.html. 
25. Myers L, Lowery M, O’Malley M, Vaughan CL. Rectification and non-linear preprocessing of EMG signals for cortico-muscular analysis. J Neurosci Methods 2003; 124: 157-65.

26. Carter GC. Coherence and time delay estimation. Proceedings of the IEEE 1987; 75: 236-255.

27. Tijssen MAJ, Munchau A, Marsden JF, Lees AJ, Bhatia KP, Brown P. Descending control of muscles in patients with cervical dystonia. Mov Disord 2002; 17: 493500 .

28. Gernert M, Richter A, Loscher W. Alterations in spontaneous single unit activity of striatal subdivisions during ontogenesis in mutant dystonic hamsters. Brain Res 1999; 821: 277-85.

29. LeDoux MS, Lorden J, Ervin JM. Cerebellectomy eliminates the motor syndrome of the genetically dystonic rat. Exp Neurol 1993; 120: 302-10.

30. Byl NN, Merzenich MM, Jenkins WM. A primate genesis model of focal dystonia and repetitive strain injury: Learning-induced dedifferentiation of the representation of the hand in the primary somatosensory cortex in adult monkeys. Neurology 1996; 47: 508-519.

31. Perlmutter JS, Tempel LW, Black KJ, Parkinson D, Todd RD. MPTP induces dystonia \& parkinsonism: clues to the pathophysiology of dystonia. Neurology 1997; 49: 1432-1438.

32. Bazzucchi I, Sbricolli P, Marzattinocci G, Felici F. Coactivation of the elbow antagonist muscle is not affected by the speed of movement in isokinetic exercise. Muscle Nerve 2006; 33: 191-99.

33. Malfait N, Sanger TD. Does dystonia always include co-contraction? A study of unconstrained reaching in children with primary and secondary dystonia. Exp Brain Res 2007; 176: 206-16. 


\section{VITA}

\section{Kunal Dilip Chaniary}

A. Personal History

Citizenship: India

B. Educational History

Virginia Commonwealth University, Richmond, Virginia

Major: Biomedical Engineering

Degree: Masters of Science

Year: 2008

Thesis: Electromyographic characterization in an animal model of dystonia

Mahatma Gandhi Mission's College, Mumbai University, India

Major: Biomedical Engineering

Degree: Bachelor's of Science

Year: 2005

C. Professional Positions

June 2004 - Dec 2004

Internship, Wipro GE Medical Systems, Mumbai, India

Installations and servicing of imaging modalities like cathlabs, CT scan, MRI and PET scanners.

D. Recent publications

Chaniary K, Baron M, Rice A, Wetzel P, Shapiro S Electromyographic

Characterization in an Animal Model of Dystonia. Mov Disord 2008 (in press) 